\title{
Intraoperative image guidance for lateral position surgery
}

\author{
Peter R. Swiatek ${ }^{1}$, Michael H. McCarthy ${ }^{2}$, Joseph Weiner ${ }^{1}$, Shivani Bhargava ${ }^{3}$, Avani S. Vaishnav ${ }^{2}$, \\ Sravisht Iyer ${ }^{2}$
}

${ }^{1}$ Department of Orthopaedic Surgery, Northwestern Memorial Hospital, Chicago, IL, USA; ${ }^{2}$ Department of Orthopaedic Surgery, Hospital for Special Surgery, New York, NY, USA; ${ }^{3}$ University of Michigan, Ann Arbor, MI, USA

Contributions: (I) Conception and design: PR Swiatek, MH McCarthy, J Weiner, S Iyer; (II) Administrative support: None; (III) Provision of study materials or patients: None; (IV) Collection and assembly of data: PR Swiatek, MH McCarthy, J Weiner, S Bhargava, AS Vaishnav; (V) Data analysis and interpretation: PR Swiatek, MH McCarthy, J Weiner, S Bhargava, AS Vaishnav; (VI) Manuscript writing: All authors; (VII) Final approval of manuscript: All authors.

Correspondence to: Peter R. Swiatek, MD. Department of Orthopaedic Surgery (Academic Office), Northwestern University Feinberg School of Medicine, 676 N. Saint Clair Street, Suite 1350, Chicago, IL 60611, USA. Email: peter.swiatek@northwestern.edu.

\begin{abstract}
Recent advances in minimally invasive spine surgery techniques have precipitated the popularity of lateral position spine surgery, such as lateral lumbar interbody fusion (LLIF) and oblique lumbar interbody fusion (OLIF). Lateral position surgery offers a unique, minimally invasive approach to the lumbar spine that allows for preservation of anterior and posterior spinal elements. Traditionally, surgeons have relied upon fluoroscopy for triangulation and implant placement. Over the last decade, intraoperative 3-dimensional navigation (ION) has risen to the forefront of innovation in LLIF and OLIF. This technology utilizes intraoperative advanced imaging, such as comminuted tomography (CT), to map the patient's 3D anatomy and allows the surgeon to accurately visualize instruments and implants in spatial relationship to the patient's anatomy in real time. ION has the potential to improve accuracy during instrumentation, decrease operating room times, lower radiation exposure to the surgeon and staff, and increase feasibility of single-position surgery during which the spine is instrumented both laterally and posteriorly while the patient remains in the lateral decubitus position. Despite the advantages of ION, the intra-operative radiation exposure risk to patients is controversial. Future directions include continued innovation in ultra low radiation imaging (ULRI) techniques and image enhancement technology and in uses of robot-assisted navigation in singleposition spine surgery.
\end{abstract}

Keywords: Intraoperative imaging; lateral position spine surgery; lateral lumbar interbody fusion (LLIF); oblique lumbar interbody fusion (OLIF); comminuted-tomography (CT) navigation; robotic spine surgery

Submitted Feb 27, 2020. Accepted for publication Jun 23, 2020.

doi: $10.21037 / \mathrm{atm}-2020$-ioi-10

View this article at: http://dx.doi.org/10.21037/atm-2020-ioi-10

\section{Introduction}

In the early 2000s, several advances set the stage for safe and pragmatic lateral access to the spine. These advances included innovations in neuromonitoring, imaging, and surgical techniques to allow for safe access to the lumbar spine through retroperitoneal fat and the psoas major muscle. Bertagnoli et al., (1) Bergey et al. (2) and Ozgur et al. (3) are largely credited with developing the lateral lumbar interbody fusion (LLIF) technique, which has been trademarked under proprietary names, including extreme lateral interbody fusion (XLIF, Nuvasive, San Diego, CA) and direct lateral interbody fusion (DLIF, Medtronic, Memphis, TN) based upon specific proprietary equipment used and the specific plane used to approach the lateral spine. An additional lateral-based procedure, the oblique lateral interbody fusion (OLIF, Medtronic, Memphis, $\mathrm{TN}$ ), is similar to the LLIF procedures, but differs in the anatomic approach to the lateral spine.

At the advent of the LLIF, pioneers of lateral-based spine surgery relied heavily upon intraoperative fluoroscopy for 
image guidance during surgery. Conventional fluoroscopy offered the benefit of being cost-effective, widely available, and familiar to surgeons. Over the last decade, computerbased navigation involving intraoperative computed tomography (CT) scan or 3D fluoroscopy have come into vogue, increasing the safety of LLIF and expanding the scope of procedures possible in lateral-based spine surgery. In this review of intraoperative image guidance for lateralposition spine surgery we will: (I) review the clinical and anatomic foundations of LLIF and OLIF; (II) explain appropriate fluoroscopic imaging techniques used during LLIF and OLIF; (III) discuss the potential applications intraoperative navigation; (IV) introduce the most recent advances in robotic spine surgery and radiation-limiting technologies.

\section{Clinical and anatomic foundations}

To understand how advances in imaging technology have improved safety, efficacy, and efficiency of LLIF and OLIF, it is vital to appreciate the anatomy of the lateral approach. The LLIF utilizes a plane through the psoas muscle, whereas OLIF approaches the spinal column anterior to the psoas. The psoas muscle arises from the transverse processes of the lumbar vertebrae and extends inferolaterally to the lesser trochanter of the femur, forming a major component of the posterior abdominal wall. The lumbar plexus, composed of the L1 to L4 nerve roots, extends directly into the posterior portion of the psoas. With the exception of the genitofemoral nerve, which arises from the L1 and L2 nerve roots, the ventral half of the vertebral column is considered safe above the L4-L5 disc space (4). The genitofemoral nerve traverses the psoas muscle and arises on the anterior surface of the L3 and L4 vertebral bodies. Injury to the genitofemoral nerve typically results in transient sensory changes in the ipsilateral scrotum and medial thigh. The femoral nerve, obturator nerve, and L4 and L5 nerve roots are at risk when approaching levels below the L4-L5 disc space and could result in more significant motor deficit if seriously injured (5). Fluoroscopic or CT-guided navigation at the start of the case and intraoperatively are imperative for identifying the target level and ensuring that the dilators and retractors used to gain access to the disk space are appropriately positioned in the safe zone ventral to the lumbar plexus.

\section{Fluoroscopic guidance}

During LLIF or OLIF, the patient is positioned in the lateral decubitus position with the iliac crest centered over the break in the bed or Wilson frame and knees slightly flexed to prevent the lumbar plexus from migrating forward within the psoas and into the dissection plane. Slight flexion is applied to the table to increase the distance between the iliac crest and rib, ultimately increasing access to the vertebral levels of interest. Fluoroscopy is used at the start of the case to obtain a true AP and true lateral. Pedicles should be clear and equidistant on the AP and endplates should be distinct on the lateral. Prior to incision, two crossing Kirschner wires are used to localize the disc space of interest. Once the surgeon has dissected down to the psoas muscle, lateral fluoroscopy is used to guide the appropriate positioning of the stimulating EMG probe and cannula in the anterior half of the disc space. In the case of an XLIF or DLIF, the probe and cannula are passed through the psoas muscle and inserted into the disc space of interest. Fluoroscopy is used to confirm the position of the probe. A guide-wire, dilators, and eventually retractors are sequentially inserted using electro-stimulation confirmation, and positioning of the final retractor construct is confirmed with fluoroscopy. After the surgeon has prepared the disc space, a trial component is inserted under biplanar fluoroscopic guidance. Once appropriate implant size is determined, the final implant is inserted and positioned under biplanar fluoroscopic guidance and retractors removed (5). Additional instrumentation, such as a lateral plate or interbody screws, may be used to strengthen the interbody construct.

Unlike posterior-based interbody procedures, lateralbased procedures require fluoroscopic, navigated or robotic assistance for screw placement in the lateral decubitus position, or intraoperative repositioning from the lateral decubitus to prone position for screw placement using a posterior approach. Although repositioning allows for adequate bilateral pedicle crew placement, repositioning is associated with increased OR time, potential anesthesia complications, and possible interbody migration (6). To avoid intraoperative repositioning, surgeons have developed techniques for minimally invasive posterior lumbar instrumentation while the patient is in the lateral position.

Blizzard et al. describe a technique using fluoroscopy to insert bilateral pedicle screws. While the patient is in the lateral position, the surgeon obtains an AP image of the target spinal levels. Percutaneous incisions are made posteriorly just lateral to the pedicles of interest. A needle and subsequently a Kirschner wire are inserted into the pedicle. The pedicles are tapped and then screws are 
inserted under AP and lateral fluoroscopic guidance.

The literature supports the safety and efficacy of fluoroscopic guidance during pedicle screw placement in single-position LLIF and OLIF. In their study of 72 patients treated with single-position LLIF or OLIF with 300 bilateral pedicle screws, Blizzard et al. recorded an overall pedicle breach rate of $5.1 \%$, which is on par with previously reported breach rates for pedicle screws inserted with the patient in the prone position (6-9). Additionally, they showed a total operative time of 88 minutes per patient, which is significantly shorter than the average of 122 minutes reported by Tohmeh et al. for cases involving repositioning and posterior instrumentation for a similar number of levels $(6,10)$. In their study directly comparing results of 66 interbody fusion procedures performed either in a single lateral position or dual position, Ziino et al. found that the dual-position group had a 44-minute longer operating time without difference in lordosis or perioperative outcomes (11). Similarly, Hiyama et al. found a 34-minute shorter operating time for the single lateral position group in their study of 30 patients (12).

\section{Intraoperative navigation}

Although fluoroscopy has traditionally been considered the intraoperative imaging standard for LLIF and OLIF, advances in intraoperative 3D CT or 3D fluoroscopy and image-guided navigation systems (IGNS) have shifted the paradigm for many spine surgeons performing lateralbased surgery. At a high-level, intraoperative imaging accurately captures the patient's anatomy in space and the IGNS software integrates the patient's anatomy with the true position of various instruments and implants used by the surgeon. This integrative map is displayed on a screen, allowing the surgeon to see a virtual representation of the operation in real-time. The advantages of image-guided navigation compared to standard intraoperative fluoroscopy have been well studied in posterior instrumentation of the spine, including increased accuracy of pedicle screw placement and decreased radiation exposure (13) for the surgeon and operating room staff $(14,15)$.

With regard to technique, image-guided navigation requires of a set of unique steps to prepare the patient for intraoperative image-acquisition and to execute instrumentation. The patient is positioned in the usual manner for a lateral-based approach to the spine: lateral decubitus, legs flexed, and either the bed slightly bent or the patients flank support by an axillary roll to open the space between the patient's ribs and iliac crest. A pin and reference arc are surgically inserted into the patient's anterior superior iliac spine (ASIS) and serves as the spatial reference point for the IGNS. An intraoperative CT or fluoroscopic image is obtained, the patient's anatomy is mapped to the IGNS, and various surgical instruments are registered to the IGNS and integrated into the virtual representation of the patient's anatomy. The dissection and disc preparation are carried out in the typical manner, however instead of using fluoroscopy for localization, the surgeon utilizes a navigated dilator, electrode, and other surgical instruments to place retractors and prepare the disc space. The trial is navigated into position and the eventually replaced with the navigated implant. Fluoroscopy is used as a final confirmation of correct implant positioning prior to closure (16).

Although no robust study has directly compared intraoperative navigation to fluoroscopy, the literature supports the safety and efficacy of navigation in LLIF. For example, Park et al. report on 8 patients treated with LLIF with a total of 22 cages. All cages were found to be placed appropriately in the anterior half or middle portions of the disc space without any sensory or motor complications post-operatively (16). In their follow-up study of 31 patients undergoing LLIF of 66 spinal levels, Joseph et al. found that $97 \%$ of cages were placed accurately with CT-based navigation. Their only observed complications included a psoas abscess in one patient that was likely unrelated to the instrumentation and transient thigh weakness and paresthesias, which are a known complication of the lateral approach (17). The last installation from Liu et al. and Park et al., described 67 patients and 117 spinal levels underwent correct cage placement in $99 \%$ of cases with approach related complications in $25 \%$ of cases (18). Surgeons have found similar success with the use of CT-based navigation and OLIF. In their case series of 49 patients and 86 levels, DiGiorgio et al. reported only seven approach-related complications and no complication related to navigation and instrumentation (19).

Few studies have investigated the utility of CT-based navigation alone, without robotic assistance, for singleposition pedicle screw placement after LLIF or OLIF. In their discussion of OLIF, Sellin et al. introduced CTnavigated percutaneous placement of pedicle screws in the lateral position (20). During this Simultaneous Lateral Interbody and Pedicle Screws (SLIPS) procedure, two surgeons work simultaneously across the table from each other. One surgeon standing anterior to the patient exposes the anterolateral spine through the oblique corridor and the 
co-surgeon stands posterior to the patient, simultaneously preparing screw entry points. Using CT-based navigation, one surgeon inserts the percutaneous pedicle screws and the other surgeon prepares the disc space. Subsequently the interbody device is navigated into place, posterior fixation is completed, and dissection corridors are closed. In their review of four patients who underwent lateral interbody fusion and posterior instrumentation using the SLIPS technique, overall pedicle breach rate was $14 \%$ with one returning to the operating room for pedicle screw replacement. The authors cite the potential for the CT-based navigation and the SLIPS technique to substantially decrease operative times and cost (20).

\section{Radiation exposure}

Surgeons and patients are at particular risk of excessive radiation exposure during most MIS spinal procedures. For example, the incidence of thyroid cancer is 25 times greater in spine surgeons compared to the general population (21). Given the limited visualization of spinal anatomy during MIS surgery, surgeons rely heavily upon imaging for safe and effective exposure and instrumentation $(22,23)$. The increased risk to surgeons and patients from radiation exposure during surgical procedures has led to the development and adoption of new technologies such as intraoperative navigation, which aim to limit radiation exposure during surgery. Given the widespread use of the standard posterior based approaches to the spine, much of the radiation research has focused on MIS posterior approaches, such as the TLIF (13) and PLIF $(24,25)$. However, recent adoption of intraoperative 3-dimensional navigation (ION) techniques in laterally-based spine surgery has spurned interest in how various imaging modalities serve to decrease radiation exposure compared to standard 2D fluoroscopy. For example, in their case series on the use of CT-based navigation for LLIF, Liu et al. found mean fluoroscopy time to be $11.7 \mathrm{~s}$ per level compared to 37.0 s per level using conventional fluoroscopy cited by Taher et al.-nearly a three-fold decrease $(18,26)$. Zhang et al. reported similar results when comparing 42 patients who underwent CT-guided OLIF versus standard OLIF using fluoroscopy (27). They showed that surgeons were exposed to $45 \mathrm{mGy}$ and fluoroscopy times of $88 \mathrm{~s}$ with conventional fluoroscopy, versus none with CT-guided navigation, as staff left the OR during the intra-op spin (27).

With regard to radiation exposure to the patient during CT-based navigation, the literature is more controversial.
For example, Zhang et al. found that the patient only received $9.38 \mathrm{mGy}$ of radiation exposure when CT was used for OLIF compared to the $45 \mathrm{mGy}$ in the fluoroscopy group (27). But this can be much higher depending upon machine setting, and can range from 6 to $66 \mathrm{mGy}$ (27). Additionally, the number of fluoroscopic images needed for safe instrumentation may be affected by a number of factors, including the approach and surgeon experience. For example, the approach in OLIF may be less direct as the trans-psoas approach in LLIF or an anterior approach in an $\operatorname{ALIF}(27)$.

With the adoption of CT-based navigation, new ultra-low radiation imaging (ULRI) protocols and image enhancement technology attempt to decrease radiation emission during MIS spine surgery. Historically, ULRI protocols alone have delivered substantial reduction in radiation emissions, but at the cost of the quality of imaging $(28,29)$. New image enhancement technologies, such as LessRay ${ }^{\circledR}$ (NuVasive, Inc., San Diego, California, USA) have been developed to sharpen images rendered via ULRI protocols by merging results of previous fluoroscopic images to render high-quality pictures. Efficacy of such technology has been documented in both the TLIF and LLIF literature $(28,30)$. Godzik et al. found a nearly $70 \%$ reduction in radiation emission during LLIF using ULRI with LessRay ${ }^{\circledR}$ image enhancement technology. Similarly, for LLIF procedures requiring posterior instrumentation, Godzik et al. found a $65.8 \%$ reduction in total radiation emission, amounting to $31.7 \mathrm{mGy}$ less radiation per screw (30).

\section{Robot-assisted surgery}

High interest in the field of robot-assisted spine surgery has led to the application of robot-assistance in LLIF and OLIF surgery, particularly pedicle screw placement during singleposition surgery. As previously discussed, several authors have described techniques for fluoroscopic $(6,11,12)$ and CT-based (20) navigated pedicle screw placement during single-position surgery. The techniques, however, have yet to be widely accepted due to technical difficulty of placing pedicle screws in the lateral position and potential risks of neurologic complication (31). The robot serves to address these concerns by assisting the surgeon with the unfamiliar trajectory of pedicle screws placed in the lateral position (32). In a series of 55 single-position LLIF cases with 328 screws placed with the robot, Huntsman et al. describes a $98 \%$ success rate with only $2 \%$ of screws being re-positioned based upon surgeon discretion. Additionally, the authors 
report no complications or mal-positioning requiring return to the operating room (33). Similarly, Khan et al. describes their success with robotic assist pedicle screw placement in the lateral position for 20 patients with 75 screws. No complications were reported and only one screw was breach to less than $2 \mathrm{~mm}$ (34).

\section{Conclusions}

Advances in imaging technology and MIS spine surgery techniques have resulted in widespread adoption of lateral position surgery, particularly LLIF and OLIF. Traditional fluoroscopic guidance for lateral-based spine procedures is gradually being supplemented or supplanted by imageguided navigation. Advantages of real-time image-guidance include increased accuracy during instrumentation and implant placement, decreased procedure time, and decreased radiation exposure to the surgeon and operating room staff. Additionally, image-guided navigation increases the feasibility and accessibility of single-position surgery during which pedicle screws are navigated from the lateral position. New frontiers in lateral position surgery include continued development of ULRI protocols and image enhancement technology, as well as advancement of robotassisted navigation for both implant and pedicle screw placement.

\section{Acknowledgments}

Funding: None.

\section{Footnotes}

Provenance and Peer Review: This article was commissioned by the Guest Editor (Dr. Sheeraz Qureshi) for the series "Current State of Intraoperative Imaging" published in Annals of Translational Medicine. The article was sent for external peer review organized by the Guest Editor and the editorial office.

Conflicts of Interest: All authors have completed the ICMJE uniform disclosure form (available at http://dx.doi. org/10.21037/atm-2020-ioi-10). The series "Current State of Intraoperative Imaging" was commissioned by the editorial office without any funding or sponsorship. SI reports personal fees from Globus Medical, Inc., personal fees from Healthgrades, other from Innovasis, outside the submitted work. The other authors have no other conflicts of interest to declare.

Ethical Statement: The authors are accountable for all aspects of the work in ensuring that questions related to the accuracy or integrity of any part of the work are appropriately investigated and resolved.

Open Access Statement: This is an Open Access article distributed in accordance with the Creative Commons Attribution-NonCommercial-NoDerivs 4.0 International License (CC BY-NC-ND 4.0), which permits the noncommercial replication and distribution of the article with the strict proviso that no changes or edits are made and the original work is properly cited (including links to both the formal publication through the relevant DOI and the license). See: https://creativecommons.org/licenses/by-nc-nd/4.0/.

\section{References}

1. Bertagnoli R, Vazquez RJ. The Anterolateral TransPsoatic Approach (ALPA): a new technique for implanting prosthetic disc-nucleus devices. J Spinal Disord Tech 2003;16:398-404.

2. Bergey DL, Villavicencio AT, Goldstein T, et al. Endoscopic lateral transpsoas approach to the lumbar spine. Spine (Phila Pa 1976) 2004;29:1681-8.

3. Ozgur BM, Aryan HE, Pimenta L, et al. Extreme Lateral Interbody Fusion (XLIF): a novel surgical technique for anterior lumbar interbody fusion. Spine J 2006;6:435-43.

4. Moro T, Kikuchi S, Konno S, et al. An anatomic study of the lumbar plexus with respect to retroperitoneal endoscopic surgery. Spine (Phila Pa 1976) 2003;28:423-8; discussion 427-8.

5. Lu Y, Wong JM, Chi JH. Lateral Lumbar Interbody Fusion: Indications sand Techniques. In: QuiñonesHinojosa A. editor. Schmidek and Sweet Operative Neurosurgical Techniques: Indications, Methods, and Results. Sixth Edition. Saunders, 2012.

6. Blizzard DJ, Thomas JA. MIS Single-position Lateral and Oblique Lateral Lumbar Interbody Fusion and Bilateral Pedicle Screw Fixation: Feasibility and Perioperative Results. Spine (Phila Pa 1976) 2018;43:440-6.

7. Smith ZA, Sugimoto K, Lawton CD, et al. Incidence of lumbar spine pedicle breach after percutaneous screw fixation: a radiographic evaluation of 601 screws in 151 patients. J Spinal Disord Tech 2014;27:358-63.

8. Amato V, Giannachi L, Irace C, et al. Accuracy of pedicle screw placement in the lumbosacral spine 
using conventional technique: computed tomography postoperative assessment in 102 consecutive patients. J Neurosurg Spine 2010;12:306-13.

9. Chapman TM, Blizzard DJ, Brown CR. CT accuracy of percutaneous versus open pedicle screw techniques: a series of 1609 screws. Eur Spine J 2016;25:1781-6.

10. Tohmeh AG, Watson B, Tohmeh M, et al. Allograft cellular bone matrix in extreme lateral interbody fusion: preliminary radiographic and clinical outcomes. ScientificWorldJournal 2012;2012:263637.

11. Ziino C, Konopka JA, Ajiboye RM, et al. Single position versus lateral-then-prone positioning for lateral interbody fusion and pedicle screw fixation. J Spine Surg 2018;4:717-24.

12. Hiyama A, Sakai D, Sato M, et al. The analysis of percutaneous pedicle screw technique with guide wireless in lateral decubitus position following extreme lateral interbody fusion. J Orthop Surg Res 2019;14:304.

13. Vaishnav AS, Merrill R, Sandhu H, et al. A Review of Techniques, Time-demand, Radiation Exposure and Outcomes of Skin-anchored Intra-operative 3D Navigation in Minimally Invasive Lumbar Spinal Surgery. Spine (Phila Pa 1976) 2020;45:E465-76.

14. Nottmeier EW, Bowman C, Nelson KL. Surgeon radiation exposure in cone beam computed tomographybased, image-guided spinal surgery. Int J Med Robot 2012;8:196-200.

15. Van de Kelft E, Costa F, Van der Planken D, et al. A prospective multicenter registry on the accuracy of pedicle screw placement in the thoracic, lumbar, and sacral levels with the use of the O-arm imaging system and StealthStation Navigation. Spine (Phila Pa 1976) 2012;37:E1580-7.

16. Park P. Three-dimensional computed tomography-based spinal navigation in minimally invasive lateral lumbar interbody fusion: feasibility, technique, and initial results. Neurosurgery 2015;11 Suppl 2:259-67.

17. Joseph JR, Smith BW, Patel RD, et al. Use of 3D CTbased navigation in minimally invasive lateral lumbar interbody fusion. J Neurosurg Spine 2016;25:339-44.

18. Liu X, Joseph JR, Smith BW, et al. Analysis of Intraoperative Cone-Beam Computed Tomography Combined With Image Guidance for Lateral Lumbar Interbody Fusion. Oper Neurosurg (Hagerstown) 2018;14:620-6.

19. DiGiorgio AM, Edwards CS, Virk MS, et al. Stereotactic navigation for the prepsoas oblique lateral lumbar interbody fusion: technical note and case series. Neurosurg
Focus 2017;43:E14.

20. Sellin JN, Mayer RR, Hoffman M, et al. Simultaneous lateral interbody fusion and pedicle screws (SLIPS) with CT-guided navigation. Clin Neurol Neurosurg 2018;175:91-7.

21. Wagner TA, Lai SM, Asher MA, et al. SRS surgeon members' risk for thyroid cancer: is it increased? Hibbs Award Nominee for Best Clinical Paper. Paper presented at: 41st Annual Meeting of the Sclerosis Research Society; 2006; Monterey, CA.

22. Bindal RK, Glaze S, Ognoskie M, et al. Surgeon and patient radiation exposure in minimally invasive transforaminal lumbar interbody fusion. J Neurosurg Spine 2008;9:570-3.

23. Grelat M, Zairi F, Quidet M, et al. [Assessment of the surgeon radiation exposure during a minimally invasive TLIF: Comparison between fluoroscopy and O-arm system]. Neurochirurgie 2015;61:255-9.

24. Tabaraee E, Gibson AG, Karahalios DG, et al. Intraoperative cone beam-computed tomography with navigation (O-ARM) versus conventional fluoroscopy (C-ARM): a cadaveric study comparing accuracy, efficiency, and safety for spinal instrumentation. Spine (Phila $\mathrm{Pa}$ 1976) 2013;38:1953-8.

25. Smith HE, Welsch MD, Sasso RC, et al. Comparison of radiation exposure in lumbar pedicle screw placement with fluoroscopy vs computer-assisted image guidance with intraoperative three-dimensional imaging. J Spinal Cord Med 2008;31:532-7.

26. Taher F, Hughes AP, Sama AA, et al. 2013 Young Investigator Award winner: how safe is lateral lumbar interbody fusion for the surgeon? A prospective in vivo radiation exposure study. Spine (Phila Pa 1976) 2013;38:1386-92.

27. Zhang YH, White I, Potts E, et al. Comparison Perioperative Factors During Minimally Invasive PrePsoas Lateral Interbody Fusion of the Lumbar Spine Using Either Navigation or Conventional Fluoroscopy. Global Spine J 2017;7:657-63.

28. Wang TY, Farber SH, Perkins SS, et al. Internally Randomized Control Trial of Radiation Exposure Using Ultra-low Radiation Imaging Versus Traditional C-arm Fluoroscopy for Patients Undergoing Single-level Minimally Invasive Transforaminal Lumbar Interbody Fusion. Spine (Phila Pa 1976) 2017;42:217-23.

29. Nayar G, Blizzard DJ, Wang TY, et al. Pedicle screw placement accuracy using ultra-low radiation imaging with image enhancement versus conventional fluoroscopy in 
minimally invasive transforaminal lumbar interbody fusion: an internally randomized controlled trial. J Neurosurg Spine 2018;28:186-93.

30. Godzik J, Nayar G, Hunter WD, et al. Decreasing Radiation Emission in Minimally Invasive Spine Surgery Using Ultra-Low-Radiation Imaging with Image Enhancement: A Prospective Cohort Study. World Neurosurg 2019;122:e805-11.

31. Xu DS, Walker CT, Godzik J, et al. Minimally invasive anterior, lateral, and oblique lumbar interbody fusion: a literature review. Ann Transl Med 2018;6:104.

32. Walker CT, Godzik J, Xu DS, et al. Minimally Invasive

Cite this article as: Swiatek PR, McCarthy MH, Weiner J, Bhargava S, Vaishnav AS, Iyer S. Intraoperative image guidance for lateral position surgery. Ann Transl Med 2021;9(1):90. doi: 10.21037/atm-2020-ioi-10
Single-Position Lateral Interbody Fusion With Robotic Bilateral Percutaneous Pedicle Screw Fixation: 2-Dimensional Operative Video. Oper Neurosurg (Hagerstown) 2019;16:E121.

33. Huntsman KT, Riggleman JR, Ahrendtsen LA, et al. Navigated robot-guided pedicle screws placed successfully in single-position lateral lumbar interbody fusion. J Robot Surg 2020;14:643-7.

34. Khan A, Meyers JE, Siasios I, et al. Next-Generation Robotic Spine Surgery: First Report on Feasibility, Safety, and Learning Curve. Oper Neurosurg (Hagerstown) 2019;17:61-9. 